However, for a Gaussian with the same mean and variance as the NB, as $n \rightarrow \infty$,

$$
\begin{aligned}
P_{N}(n)= & \exp \left[-\left(n-\left\langle n_{t h}\right\rangle\right)^{2} /\left(2\left\langle n_{t h}\right\rangle^{2}\right)\right] /\left[\sqrt{ }(2 \pi)\left\langle n_{t h}\right\rangle\right) \\
& \propto \exp \left(-n^{2}\right)
\end{aligned}
$$

Thus, the right tails of the two distributions differ in form, as is readily seen in Fig. 2.

As is also evident in Fig. 2, as $n \rightarrow 0$ the left tail of the NNB distribution decays more rapidly than the left tail of the equivalent Gaussian. Indeed for $n=0, P_{S N}(0)$ for the NNB is

$$
\begin{aligned}
P_{S N}(0) & =\exp \left[-g\langle N\rangle /\left(1+\left\langle n_{t h}\right\rangle\right)\right] /\left(1+\left\langle n_{t h}\right\rangle\right)^{M} \\
& \approx \exp (-3 b\langle N\rangle)\left[\exp (-b\langle N\rangle) /\left\langle n_{t h}\right\rangle^{M}\right]
\end{aligned}
$$

with $b=g /\left(4\left\langle n_{t h}\right\rangle\right)$, whereas for a Gaussian with the same mean $g\langle N\rangle$ and variance $2 g\langle N\rangle\left\langle n_{t h}\right\rangle$ as the NNB (assuming $\langle N\rangle$ very large),

$$
\begin{aligned}
P_{S N}(0) & =\left[\langle N\rangle^{-1 / 2} /\left(4 \pi g\left\langle n_{t h}\right\rangle\right)^{1 / 2}\right] \exp \left[-g\langle N\rangle /\left(4\left\langle n_{t h}\right\rangle\right)\right] \\
& \approx\langle N\rangle^{-1 / 2}\left[\exp (-b\langle N\rangle) /\left(4 \pi g\left\langle n_{t h}\right\rangle\right)^{1 / 2}\right] .
\end{aligned}
$$

Thus, even as $\langle N\rangle$ becomes very large, the left tail of the NNB cannot be approximated by a Gaussian.

\section{UNIFORM CNR DESIGN RULES FOR COHERENT SUBCARRIER MULTIPLEXED SYSTEM WITH MULTIOCTAVE FREQUENCY ALLOCATION}

Indexing terms: Multiplexers and multiplexing. Phase modulation

For a CSCM system with multioctave configuration, the CNR difference among channels is significant and needs to be taken into consideration. Here we take equal optimal be taken into consideration. Here we take 'equal optimal CNR of the first and central channels' as a criterion, then we derive a design rule to reduce CNR difference significantly. with lower received signal power to achieve the same CNR requirement.

Introduction and system description: For a coherent subcarrier multiplexed (CSCM) system as shown in Fig. 1, the degree of nonuniform CNR in a multioctave configuration may be large as reported in Reference 1. Usually in a multioctave system,

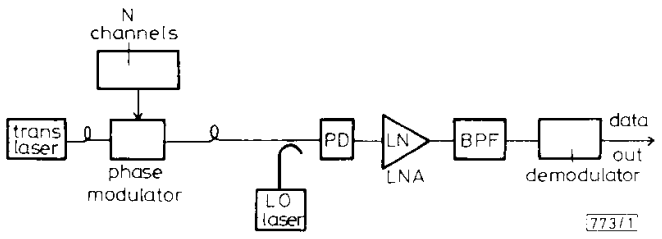

Fig. 1 System block diagram of CSCM

the second-order intermodulation $\left(I M D_{2}\right)$ contaminates the first channel mostly, but the third-order intermodulation $\left(I M D_{3}\right)$ contaminates the central channel mostly. ${ }^{1}$ In this letter, we first obtain the optimal CNR expressions of both the first and the central channel and use the criterion 'equal optimal CNR of the first and central channels' to equalise the CNR performance approximately. Then we can obtain the phase modulation (PM) index in terms of channel spacing, the total number of channels, and the 'octave-number' with corre sponding received power to meet the CNR requirement of the first channel (worst case in a multioctave system). The optical
CSCM system consists of $N$ equispaced channels with signal bandwidth $B$ and channel separation $\Delta f$. To reduce $I M D_{2}$, we locate the frequency of the $i$ th channel $(i=1, \ldots, N)$ at $f_{i}=$ $(i-1) \Delta f+F_{\min } \Delta f+\Delta f / 2$, where $F_{\text {min }}$ is an integer. The offset frequency $\Delta f / 2$ is employed to let $I M D_{2}$ degrade the channel signal least. The power spectra of $I M D_{2}$ and $I M D_{3}$ can be taken as the convolution of the power spectrum of each channel $^{2}$ and their magnitudes are determined by the signal power level and the phase modulation (PM) index

Derivation of CNR and optimum PM index: The CNR for large local oscillator power which suppresses the thermal noise can be derived by using the first-order approximations of $J_{0}(\beta)$ and $J_{1}(\beta)$ as

$C N R=\left(4 q B /\left(R P_{S} \beta^{2}\right)+h_{2} K_{2} \beta^{2} / 4+h_{3} K_{3} \beta^{4} / 16\right)^{-1}$

where $q$ is the electron charge, $R$ is the photodiode responsivity, $\boldsymbol{P}_{S}$ is the received signal power, and $\beta$ is the PM index (assumed equal for all channels). $h_{2}$ and $h_{3}$, related to the power spectra of $I M D_{2}$ and $I M D_{3}$ in the neighbourhood of the signal band, are the fractions of the power within the passband of the bandpass filter. Under the condition of equal channel spacing, the value of $h_{2}$ for the ideal rectangular signal spectrum can be expressed as $h_{2}=(3-\Delta f / B)^{2} / 8$ for $\Delta f<3 B$, and $h_{2}=0$ for $\Delta f \geq 3 B$. The value of $h_{3}$ for the ideal rectangular signal spectrum is $2 / 3 . K_{2}(i)$ and $K_{3}(i)$ represent the numbers of $I M D_{2} s$ and $I M D_{3} s$ contaminating the $i$ th channel, respectively. They can be expressed for a multioctave configuration as in References 3 and 4 , case $(A)(1<X \leq 2)$ : $K_{2}(i)=N(1-1 / X)-i+1$ for $1 \leq i \leq N-F_{\min }, K_{2}(i)=0$ for $N-F_{\min }+1 \leq i \leq F_{\min }+1$ and $K_{2}(i)=(i-N / X-1) / 2$ for $F_{\min }+2 \leq i \leq N ; \operatorname{case}(B)(2<X): K_{2}(i)=N(1-1 / X)-i$ +1 for $1 \leq i \leq F_{\min }+1, K_{2}(i)=[N(2-3 / X)-i+1] / 2$ for $F_{\min }+2 \leq i \leq N-F_{\min }$ and $K_{2}(i)=(i-N / X-1) / 2$ for $N$ $-F_{\min }+1 \leq i \leq N$. For both cases $(A)$ and $(B), K_{3}(i)=i(N$ $-i+1) / 2+\left[(N-3)^{2}-5\right] / 4$, where $X \equiv N / F$, the 'octave-number') and $X=2,3, \ldots$, etc. represent the two-, three-, .., octave configuration.

Here we define the CNR difference, $\Delta(i, j)$, between channels $i$ and $j$ from eqn. 1 as

$$
\begin{aligned}
\Delta(i, j) & =10 \log _{10} \frac{C N R(i)}{C N R(j)} \\
& =10 \log _{10} \frac{1+\left[h_{2} K_{2}(j)+h_{3} K_{3}(j) \beta^{2} / 4\right] /\left[16 q B /\left(R P_{S} \beta^{4}\right)\right]}{1+\left[h_{2} K_{2}(i)+h_{3} K_{3}(i) \beta^{2} / 4\right] /\left[16 q B /\left(R P_{S} \beta^{4}\right)\right]}
\end{aligned}
$$

We can obtain the optimal PM index that maximises the CNR of the $i$ th channel as $\beta_{\text {opt }}=\left\langle 0 \cdot 5\left\{-y+\sqrt{ }\left[y^{2}+\right.\right.\right.$ $\left.\left.\left.\left(64 / 3 h_{3} K_{3}(i) C N R\right)\right]\right\}\right\rangle^{1 / 2}$, where $y=\left[8 h_{2} K_{2}(i)\right] /\left[3 h_{3} K_{3}(i)\right] .^{1}$ The corresponding receiver sensitivity is $P_{S}=(4 q B / R)$, $\left[\beta_{o p t}^{2} / C N R-h_{2} K_{2}(i) \beta_{o p t}^{4} / 4-h_{3} K_{3}(i) \beta_{o p t}^{6} / 16\right]$. Then we can express this maximum obtainable CNR for this channel in terms of $\beta_{o p t}$ as $C N R_{o p t}=\left[h_{2} K_{2}(i) \beta_{o p t}^{2} / 2+3 h_{3} K_{3}(i) \beta_{o p t}^{4} / 16\right]^{-1}$.

The design rules: We usually have the same received power $\boldsymbol{P}_{S}$ and unified system PM index $\beta_{s y s}$ for all the channels in the CSCM system with total $N_{s y s}$ channels. We also need to keep the CNR of all channels above a specific value. Therefore, we may choose the appropriate $P_{S}, \beta_{s y s}$ and $\Delta f$ to meet the requirement. Here, we are concerned about uniform CNR for all channels' and will solve this problem as follows: first, we take channels 1 and $N_{s y .} / 2$ as the worst channels under the consideration of $I M D_{2}$ and $I M D_{3}$, respectively. We apply 'equal optimal CNR of channels 1 and $N_{s y s} / 2$ ' as a criterion, that is, $C N R_{o p t}(1)=C N R_{\text {opt }}\left(N_{\text {sys }} / 2\right)$, to obtain the corresponding $\beta_{s y s}=2(3-\Delta f / B)\left[K_{2}(1)-K_{2}\left(N_{s y s} / 2\right) / N_{s y s}\left(N_{s y s}-2\right)\right]^{0.5}$ Then we may simplify it as $\beta_{s y s}=2(3-\Delta f / B)\left(1-1 / X / N_{s y s}\right.$ $-2)^{0.5}$ (case $\left.(A)\right)$, and $\beta_{s y s}=(3-\Delta f / B)\left[(1+2 / X) / N_{s y s}\right.$ $-2)]^{0.5}+0.016$ (case $(B)$ ). Hence, the unified system PM index can be obtained from the given total channel number and octave-number; together with the specified channel spacing $\Delta f$ to achieve the required CNR value with the corresponding $P_{S}$

ELECTRONICS LETTERS 28th March 1991 Vol. 27 No. 7 
Numerical examples and conclusion: Consider a multioctave system with digital data rate $100 \mathrm{Mbit} / \mathrm{s}(B=100 \mathrm{MHz})$ and $R=1 \mathrm{~A} / \mathrm{W}$. Here we consider the two- and four-octave systems (that is, $X=2$ and 4 ) with total channel number $N_{s y s}=40$. We may find that the $\beta_{\text {sys }}$ and $\beta_{o p t}$ of channels 1 and $N_{s y s} / 2$ cross at some specified CNR with corresponding channel spacing $\Delta f$ as shown in Fig. 2. Table 1 lists the operating conditions proposed here $(A, B, C, D)$ and in Reference 1 $(E, F, G, H)$. We obtain the CNR difference $\Delta(i, 1)$ between the first and ith channels against the numerical channel positions as shown in Fig. 3 with the corresponding operating condi-
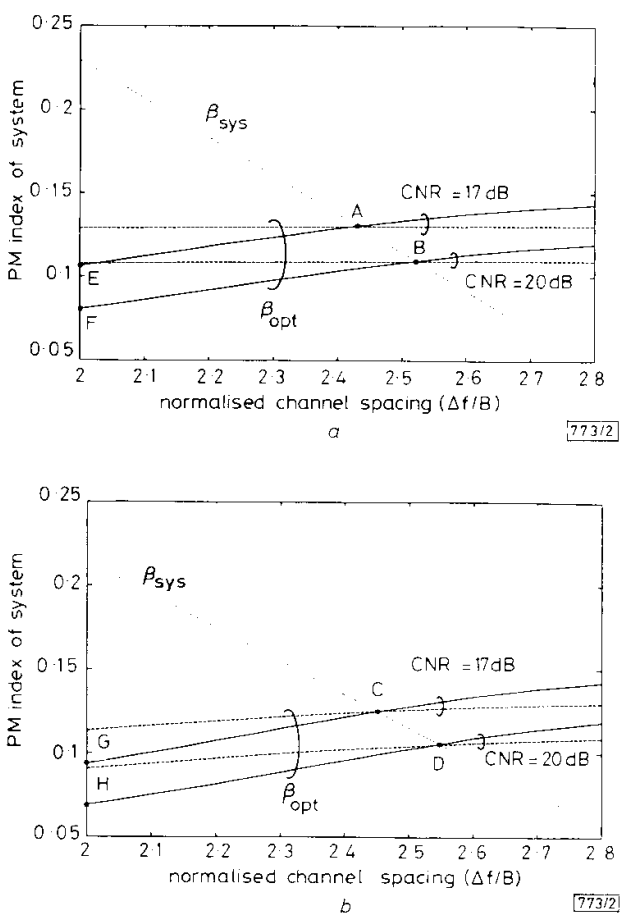

Fig. $2 P M$ index of channels $I, N_{s y s} / 2$ and system against the normalised channel spacing with $N_{s y s}=40, B=100 \mathrm{MHz}$

$a$ Case $(A): \mathrm{X}=2$

$b$ Case $(B): X=4$

- channel 1

- - - channel $N_{s y y} / 2$

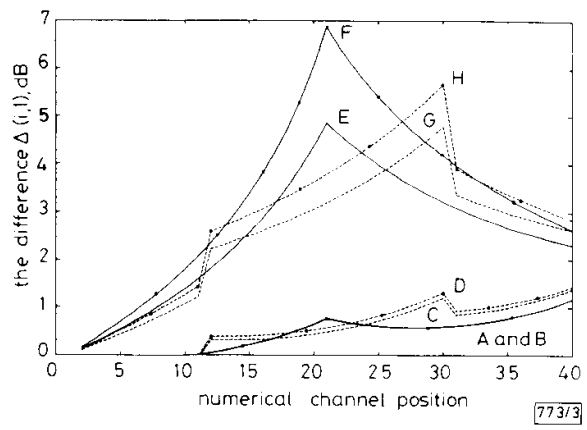

Fig. $3 C N R$ difference $\Delta(i, I)$ between channel $i$ and I against the numerical channel position for the operating conditions in Table $l$ with $N_{s y s}=40$ and $B=100 \mathrm{MHz}$

$\mathrm{CNR}=17 \mathrm{~dB}$

Case $(A)$
$-\ldots$ Case $(B)$
CNR $20 \mathrm{~dB}$
- Case $(A)$
- Case $(B)$ tions in Table 1. As shown in Reference 1, the CNR difference may be large for fixed channel spacing $(\Delta f=2 B)$ in a multioctave system when only the first channel's optimisation is

Table 1 OPERATING CONDITIONS

\begin{tabular}{|c|c|c|c|c|}
\hline & $\beta_{s y s}$ & $\Delta f / B$ & $C N R(\mathrm{~dB})$ & $P_{s}(\mathrm{dBm})$ \\
\hline \multicolumn{5}{|c|}{ This letter } \\
\hline$A$ & $0 \cdot 1286$ & $2 \cdot 43$ & 17 & $-34 \cdot 3$ \\
\hline$B$ & 0.1087 & 2.52 & 20 & $-29 \cdot 8$ \\
\hline$C$ & $0 \cdot 1261$ & $2 \cdot 45$ & 17 & $-33 \cdot 8$ \\
\hline$D$ & $0 \cdot 1065$ & $2 \cdot 55$ & 20 & $-29 \cdot 4$ \\
\hline \multicolumn{5}{|c|}{ Reference 1} \\
\hline$E$ & $0 \cdot 1065$ & 2 & 17 & $-32 \cdot 5$ \\
\hline$F$ & $0 \cdot 0804$ & 2 & 20 & $-27 \cdot 0$ \\
\hline$G$ & 0.0935 & 2 & 17 & $-31 \cdot 4$ \\
\hline$H$ & 0.0695 & 2 & 20 & $-25 \cdot 7$ \\
\hline
\end{tabular}

considered. Given the octave-number and the total channel number, we can select the unified system PM index $\beta_{\text {sys }}$ to achieve an approximately uniform CNR. Finally, we can choose the required channel spacing to achieve a given CNR value with the corresponding received signal power. Therefore, we can reduce the CNR difference from 7 to $1.5 \mathrm{~dB}$ with lower received power to achieve the same CNR requirement.

Y.-H. LEE

J. WU

13th December 1990

H.-W. TSAO

Department of Electrical Engineering

National Taiwan University

Taipei, Taiwan, Republic of Chine

\section{References}

1 GRoss, R., and OLSHANSKY, R.: 'Multichannel coherent FSK experiments using subcarrier multiplexing techniques', J. Lightwave Technol., 1990, 8, (3), pp. 406-415

2 STEPHENS, W. E., and JOSEPHS, T. R.: 'A $1.3 \mu \mathrm{m}$ microwave fiber optic link using a direct-modulated laser transmitter', J. Lightwave Technol., 1985, 3, pp. 308-315

3 OLShanSKY, R., LANZiSERA, V. A., and hill, P.: 'Subcarrier multiplexed lightwave systems for broadband distribution', J. Lightwate Technol., 1989, 7, pp. 1329-1341

4 ABUELMA'ATTI, M. T.: 'Carrier-to-intermodulation performance of multiple FM/FDM carriers through a GaAlAs heterojunction laser diode', IEEE Trans., 1985, COM-33, (3), pp. 246-248

\section{THROUGHPUT ANALYSIS OF CDMA WITH DPSK MODULATION AND DIVERSITY IN} INDOOR RICIAN FADING RADIO CHANNELS

Indexing terms: Digital communication systems, Radio links

The throughput of a slotted CDMA system with DPSK modulation is derived, considering selection diversity and maximum ratio, combined in an indoor Rician fading channel. Computational results are obtained for typical values of maximum RMS delay spread and data rates. The values of maximum RMS delay spread and data rates. The
effect of $(15,7) \mathrm{BCH}$ code on the throughput is also investigated.

Introduction: We present a throughput analysis of slotted code division multiple access (CDMA) for indoor wireless communications with a differential phase shift keying (DPSK) modulation scheme and two forms of diversity methods (antenna selection diversity and maximum ratio combining) to combat the multipath fading in indoor radio channels. The 